\title{
Neural Network for Modeling the Mechanical Properties of Gelatin-Cellulose Nanocrystals Hydrogel Membrane for Heavy Metal ions Removal from Wastewater
}

\author{
John Kabuba ${ }^{1 *}$ \\ *Department of Chemical and Metallurgical Engineering, Vaal University of Technology, Private Bag \\ X021, South Africa
}

\begin{abstract}
The mechanical properties of Gelatin-cellulose nanocrystals hydrogel membrane were investigated for the removal of heavy metal ions from wastewater. The membrane was characterized using Scanning Electron Microscopy (SEM) and Fourier Transform Infrared Spectroscopy (FTIR) analysis. Neural Network ( $\mathrm{NN}$ ) model was developed to predict the mechanical properties such as Young's modulus, tensile strength, and elongation. The NN predicted results are very close to the experimental results with $\mathrm{R}^{2}=0.99315$. The predicted values were found to be in excellent agreement with the experimental data and the current model has a good learning precision and generalization. The results revealed that the developed model is very accurate.
\end{abstract}

\section{Introduction}

Heavy metals in wastewater are highly toxic, not biodegradable and effortlessly accumulated in living organisms [1]. They can be the origin of illnesses such as cancer, high blood pressure, nervous system diseases and kidney failure at elevated concentrations [2]. The removal of heavy metal ions from wastewater is mandatory to protect the population and the environment. Various wastewater treatment technologies including convention filtration, coagulation-flocculation, reverse osmosis and adsorption have been introduced using membranes [3]. According to Singh and Hankins [4], the use of membrane in the removal of heavy metal ions from wastewater is economic, environmentally friendly and easily accessible. The varying nature and complexity of wastewater requires the improvements, in terms of quality of permeate and efficiency to enhance the reduction in membrane fouling. Nowadays, various researchers are focused on solving this problem by obtaining a new material applicable in any wastewater treatment facilities. Proteins are natural polyelectrolytes and have attracted great interest due to their ability to form self-assembled structures [5]. Gelatin is a protein, commercially available, biodegradable and presents good processability and film-forming properties [6]. Gelatin films present moisture-sensitive and exhibit low water vapor barrier and mechanical resistance under high relative humidity due to the hydrophilicity of the polypeptide chains [7]. Cellulose nanocrystals (CNCs) were used

*Corresponding author: johnka@vut.ac.za 
to overcome these limitations due to their hydrophilic, renewable and non-toxic characteristic [8]. Several researchers have described a joint synergistic effect between gelatin and CNCs toward the formation of percolated networks stabilized by hydrogen bonding. The work done by George and Siddaramaiah [9] showed that the use of CNCs, obtained from cellulose maximized the tensile strength, Young's modulus, and stiffness of gelatin films. Leite, Ferreira, Correa, Moreira, and Mattoso [10] demonstrated that adding CNCs increased the tensile strength and high elastic modulus and low thermal expansion coefficient. Tensile strength and elongation at break play an important role in determining the flexibility and strength of a film specially to overcome the external stress that occurs during the process of heavy metals removal in wastewater. The estimation of mechanical properties is a very promising method as there is a growing interest in minimizing the quality control and conducting tests with real-time prediction. Neural networks (NN) have been found to be one of the most promising numerical simulation techniques to make reliable predictions [11]. Recently, several studies have reported the successful implementation of NNs for data modelling for a wide range of engineering applications [12]. $\mathrm{NN}$ is a system of data processing established on the structure of a biological neural system [13]. Even though there are numerous studies focusing on the development of $\mathrm{NN}$ models for predicting the properties of membrane system, not much research has been reported on the application of the $\mathrm{NN}$ models to predict the mechanical properties of gelatin-cellulose nanocrystals hydrogel membranes.

\section{Materials and methods}

\subsection{Preparation of the gelatin-cellulose nanocrystals hydrogen membrane and metal solution}

All the chemicals and reagents used in this work were analytical reagent grade purchased from Sigma-Aldrich, South Africa. Adequate quantities of CNCs and gelatin (purity $\geq 98 \%$, $0.5 \mathrm{wt} \%$ and $5.0 \mathrm{wt} \%$ on a dry gelatin mass basis) were supplied by Council for Scientific and Industrial Research (CSIR), Pretoria, South Africa and dispersed in $50 \mathrm{~mL}$ of water. The CNCs suspension was then homogenized using a homogenizer to ensure the CNCs suspended uniformly. Certain amount of gelatin was then added into CNCs suspension. The mixture was then stirred at $55^{\circ} \mathrm{C}$ until a homogeneous viscous mixture was obtained. The crosslinking agent (EDTA 1\%) was then added drop wise. After 4 hours, the mixture was poured into a petri dish and placed in oven at $45^{\circ} \mathrm{C}$ until the mixture was dried. Hydrogels as films were removed from the petri dish and washed with distilled water to remove unreacted chemicals. The unreacted chemicals have been removed from hydrogel using acetone. Heavy metal ions were prepared by dissolving copper sulfate $\left(\mathrm{CuSO}_{4} \cdot 5 \mathrm{H}_{2} \mathrm{O}\right)$, nickel nitrate $\left(\mathrm{NiNO}_{3}\right)_{2} \cdot 6 \mathrm{H}_{2} \mathrm{O}$ and lead chloride $\left(\mathrm{PbCl}_{2}\right)$ for feed solution for testing the membrane performance. The initial $\mathrm{pH}$ of the solution was adjusted with $0.1 \mathrm{M} \mathrm{HCl}$ or $0.1 \mathrm{M} \mathrm{NaOH}$. All experiments were carried out in triplicate and the results were reported as an average value at $95 \%$ confidence level.

\subsection{Characterization of the membrane}

\subsubsection{Determination of Zeta-potential $(\mathrm{pHz})$}

The Zeta-potentials $(\mathrm{pHz})$ provide of the net charges on particle surfaces as a function of $\mathrm{pH}$. Zeta-potentials of aqueous solution of CNCs dispersion and gelatin were obtained at $\mathrm{pH} 4,6$ and $8\left(0.5 \mathrm{wt} \% \mathrm{CNCs}\right.$ on a dry gelatin mass basis). Zetasizer Malvern Nano ZS at $25^{\circ} \mathrm{C}$ was used for the charge on the membrane surface. The sample was mixed with a sonicator for 5 min with microtip before analysis. All the measurements were performed in triplicate. 


\subsubsection{Mechanical tests}

The mechanical tests were carried out in an Instron Universal Testing Machine equipped with $100 \mathrm{~N}$ load cell at room temperature. The Gelatin-CNCs hydrogel membrane samples were stretched using crosshead speed of $10 \mathrm{~mm} / \mathrm{min}$ with clamps initially separately by $100 \mathrm{~mm}$. Tensile strength, Young's modulus, and elongation were calculated from the stress-strain curves. The tensile tests were performed with five replicates per membrane samples.

\subsubsection{Characterization analysis}

The characterization of gelatin-CNCs hydrogel membranes was done using Fourier transform Infra-Red (FT-IR) spectroscopy and scanning electron microscopy (SEM). Perkin Elmer Spectrum 100 spectrometer on $\mathrm{CaF}_{2}$ plates was used for FT-IR spectroscopic analysis and recorded in the 500-4000 $\mathrm{cm}^{-1}$ range at a resolution of $4 \mathrm{~cm}^{-1}$. SEM analysis using JEOL JSM-840 Field Emission Scanning Emission Microscope instrument was used for the morphology and composition of the materials were characterized, running at $2 \mathrm{kV}$ accelerating voltage. The quantity of membrane used was between $0.5-1.0 \mathrm{~g}$ and backfilled with nitrogen gas (99\% High Purity) and subsequently outgassed at $25^{\circ} \mathrm{C}$ for $24 \mathrm{hrs}$ to remove adsorbed impurities acquired during ambient exposure. The Thermogravimetric analysis (TGA) was done using a Q500 equipment. A mass of $10 \mathrm{mg}$ of sample was taken in a standard Ptcrucible and heated from 25 to $600^{\circ} \mathrm{C}$ with heating rate of $10^{\circ} \mathrm{C} \mathrm{min}{ }^{-1}$, under inert atmosphere of nitrogen $(60 \mathrm{~mL} / \mathrm{min})$. Samples before the TGA tests were conditioned in a ventilated climatic chamber at $23 \pm 1{ }^{\circ} \mathrm{C}$ and $50 \pm 5 \% \mathrm{RH}$ for $48 \mathrm{hrs}$.

\subsubsection{Heavy metal ions removal}

The heavy metal ions removal tests were made for $\mathrm{Cu}^{2+}, \mathrm{Ni}^{2+}$ and $\mathrm{Pb}^{2+}$. The removal tests were done with solutions containing $200 \mathrm{mg} / \mathrm{L}$ for each heavy metals. The $\mathrm{pH}$ of the solution was 6.5 and for a solution containing $30 \mathrm{mg} / \mathrm{L}$ of each heavy metal where the $\mathrm{pH}$ value was adjusted to 3 . The samples were left on a shaking board at $25^{\circ} \mathrm{C}$ for $24 \mathrm{hrs}$ after which the hydrogels were removed from the solution. The heavy metal mass concentration in each solution before and after removal was measured using Atomic Absorption Spectroscopy (AAS, thermos electrical corporation 3300). All heavy metal solutions were diluted prior to the measurements. The heavy metal ion removal percentage was calculated using Equation (1).

Heavy metal ion removal $(\%)=\frac{C_{o-} C_{e}}{C_{o}} \times 100$

where $C_{e}$ and $C_{o}$ is the mass concentration $(\mathrm{mg} / \mathrm{L})$ of heavy metal remaining after and the initial in the solution, respectively.

\subsection{Neural Network modelling development}

The Neural Network Toolbox V4.0 MATLAB, 2020a mathematical software was used for modelling the mechanical properties of gelatin-cellulose nanocrystals hydrogel membrane. In this study, 120 data sets were used to develop the $\mathrm{NN}$ model. The input variables were Young's modulus, tensile strength and elongation. The corresponding heavy metal ions removal (\%) was used as target. A three-layered backpropagation NN with tangent sigmoid transfer function (tansig) at hidden layer and a linear transfer function (purelin) at output layer were used. The backpropagation algorithm was used for the network training. The number of hidden neurons was fixed in order to study the influence of the input variables to optimize the selection of the wastewater treatment parameters which have to be taken into account for membrane prediction. The connection between the neurons is assigned with a weight to each neuron, is added to the sum of the weighted inputs. Moreover, an activation function is defined for each layer of the NN. The activation functions are specific to a layer 
and can be different for the hidden and output layers. In order to fit the demands of the activation functions, inputs and targets of the NN are normalized between 0 and 1 . The normalization function is given in the Equation (2).

$Z=\frac{X-X_{\min }}{X_{\max }-X_{\min }}$

where $Z$ is the normalized input, $X$ is the original input and $X_{\min }$ and $X_{\max }$ are the minimum and maximum values, respectively of the input variables over the whole range of input values of the dataset. The training phase of $\mathrm{NN}$ which is randomly defined to minimize its performance factor defined as the mean squared error (MSE). The learning procedure initialised the random initial weights and biases for the $\mathrm{NN}$ and applied the LevenbergMarquart (LM) algorithm to minimise the MSE. The mean squared error is allows to compare the outputs given by the NN and the outputs measured during the experiment. The MSE is given in the Equation (3).

$M S E=\frac{1}{N} \sum_{N}^{i=1}\left(Y^{(i)}-Y_{t}^{(i)}\right)^{2}$

where $Y^{(i)}$ and $Y_{t}^{(i)}$ are the $i^{\text {th }}$ predicted and target outputs, respectively over $\mathrm{N}$ data.

The validation dataset is used to avoid overfitting of the network and another part of the dataset is used to test the network after the training phase and evaluate its accuracy [12]. The performance of the NN can be evaluated using the root-MSE (RMSE) along with the coefficient of determination $\left(R^{2}\right)$ and the relative error presented in Equations (4) and (5), respectively.

$R^{2}=\frac{1-\sum_{N}^{i=1}\left(Y_{t}^{(i)}-Y^{(i)}\right)^{2}}{\sum_{N}^{i=1}\left(Y_{t}^{(i)}-\hat{Y}\right)^{2}}$

$\operatorname{Error}(\%)=\left|\frac{Y_{t}^{(i)}-Y^{(i)}}{Y_{t}^{(i)}}\right| \times 100$

where $\hat{Y}$ is the average of the predicted outputs over the $\mathrm{N}$ data. In order to check the generality of the network prediction and to prevent the data overfitting, the data sets were divided into training, validation and test dataset with the percentage of 70, 15 and 15, respectively. Data were subject to analysis of variance (ANOVA). The mean values were tested at a confidence level of $95 \%$.

\section{Results and Discussion}

\subsection{Physical and rheological properties of Gelatin-Cellulose Nanocrystals Hydrogel Membrane}

Figure 1 illustrates the evolution of the Zeta-potential of gelatin solution and CNCs suspension upon $\mathrm{pH}$ variation. The isoelectric point $(\mathrm{pI})$ of gelatin solution occurred at around $\mathrm{pH} 6$, at which a $\mathrm{pHz}=0$. Gelatin has the ability to present different charges depending on the $\mathrm{pH}[8]$. At $\mathrm{pH}<\mathrm{pI}$, the gelatin solution showed a net positive charge, which was attributed to the protonation of the amino groups, while at $\mathrm{pH}>\mathrm{pI}$ the gelatin solution showed a net negative charge, due to ionization of the carboxyl groups. For the CNCs suspension, the $\mathrm{pHz}$ value was found to be negative regardless of the $\mathrm{pH}$. The $\mathrm{pHz}(<-40 \mathrm{mV}$ and $>+40 \mathrm{mV})$ could maintain a stable colloidal system due to the repelling forces between particles. In aqueous solutions, electrostatic charges are one of the main driving forces for interactions between charges biopolymers [7]. 


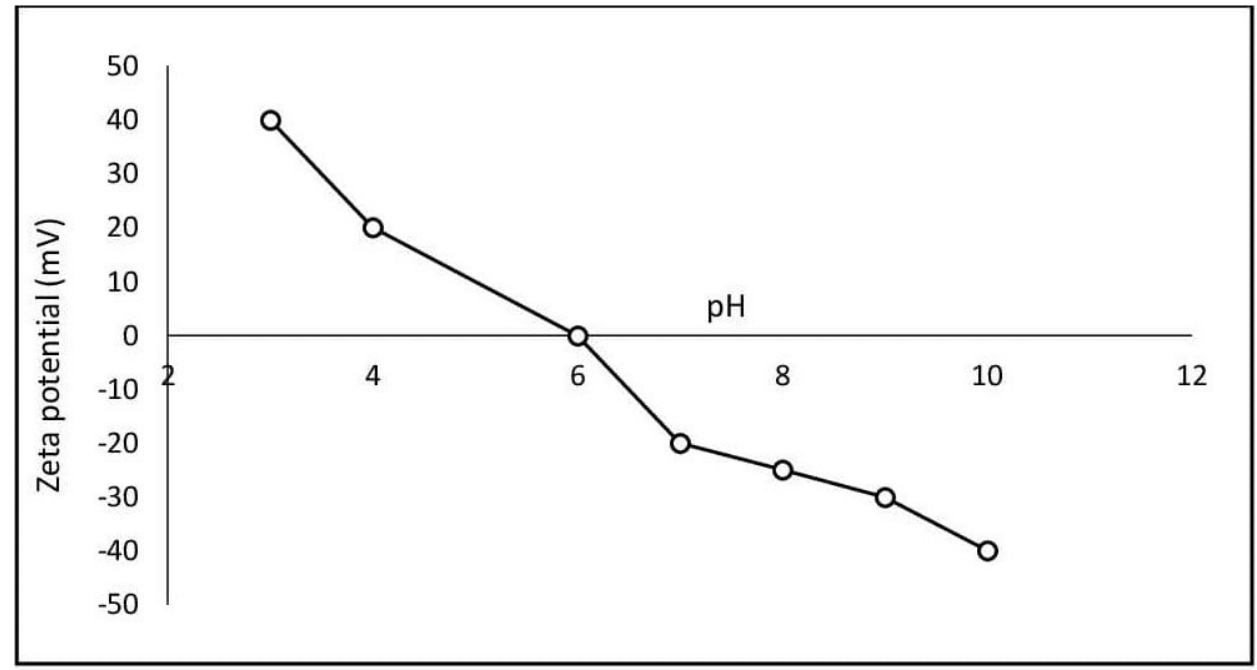

Fig. 1. Effect of $\mathrm{pH}$ on zeta potential

\subsection{Characterization of Gelatin-Cellulose Nanocrystals Hydrogel Membrane}

\subsubsection{FTIR analysis}

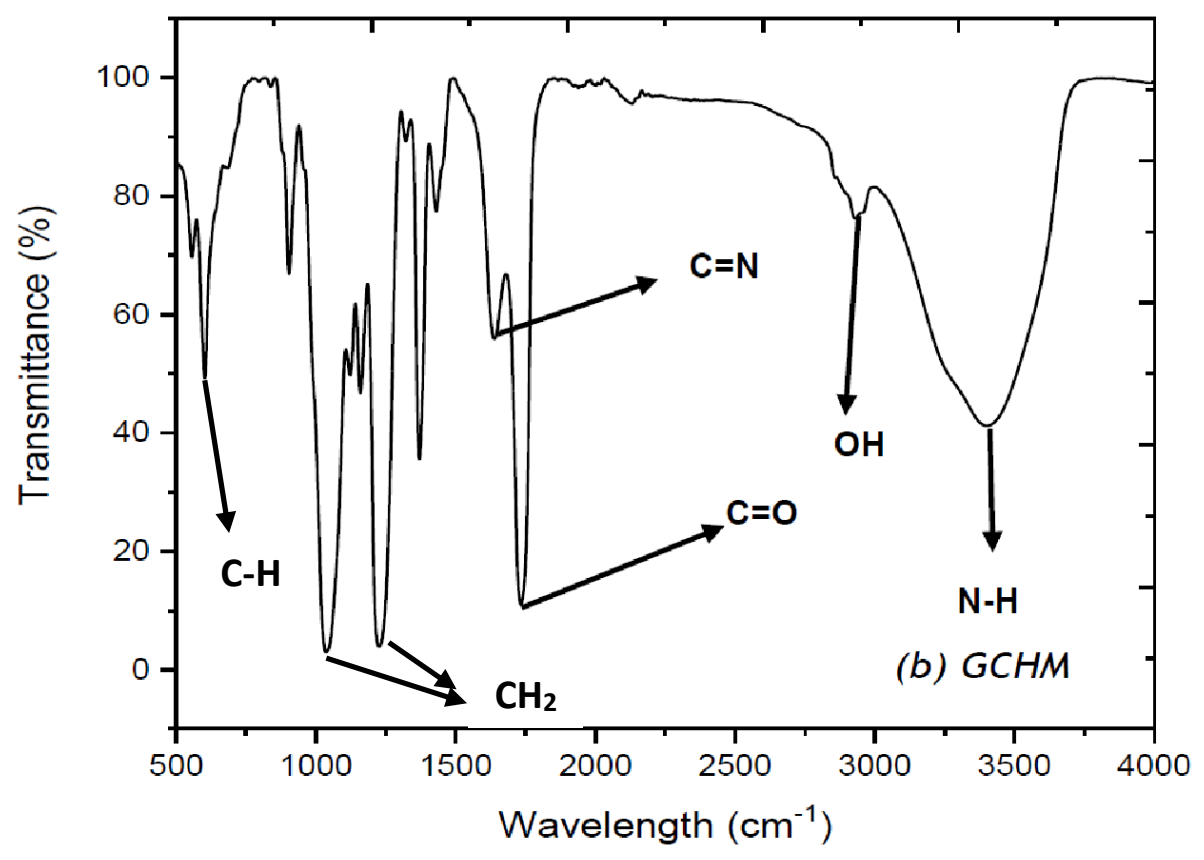

Fig. 2. FT-IR spectra of the Gelatin-cellulose Hydrogel Membrane

The FT-IR analysis of gelatin and CNCs membrane is presented in Figure 2. The FT-IR spectra were used to confirm the crosslinking of the gelatin chain and to study the changes that occurred in the functional groups of the gelatin following the reaction with bacterial cellulose. Gelatin-CNCs membrane has shown the characteristic bands around 2500 and $3000 \mathrm{~cm}^{-1}$ that represents the strong elongation vibration band of the $\mathrm{OH}$ function. The band around $1450 \mathrm{~cm}^{-1}$ is the vibration shear of $\mathrm{CH}_{2}$ and it is regarded as crystallinity band in any 
cellulosic material [13]. The peak at $1700 \mathrm{~cm}^{-1}$ is characteristic of the elongation vibration band of the carboxyl $(\mathrm{C}=\mathrm{O})$ confirmed by the characteristic band around $890 \mathrm{~cm}^{-1}$ that corresponds to the $\mathrm{C}-\mathrm{H}$ of aldehyde group [13]. The amide is represented by the deformation vibration peaks of $\mathrm{N}-\mathrm{H}$ at $3500 \mathrm{~cm}^{-1}$. Moreover, the amide is characterized by the elongation vibration of $\mathrm{C}=\mathrm{O}$ to which is added a contribute band of elongation vibration of $\mathrm{C}-\mathrm{N}$. The peaks below $1000 \mathrm{~cm}^{-1}$ are characteristic of low molecular weight amides [14]. The incorporation of CNCs on the membrane is confirmed by the peak at $3500 \mathrm{~cm}^{-1}$, which is attributed to the stretching vibration of hydroxyl groups from CNCs. In addition, a higher intensity of this peak is observed when increasing the CNCs content. This occurrence can contribute to the increased hydrophilicity of the membrane.

\subsubsection{SEM analysis}

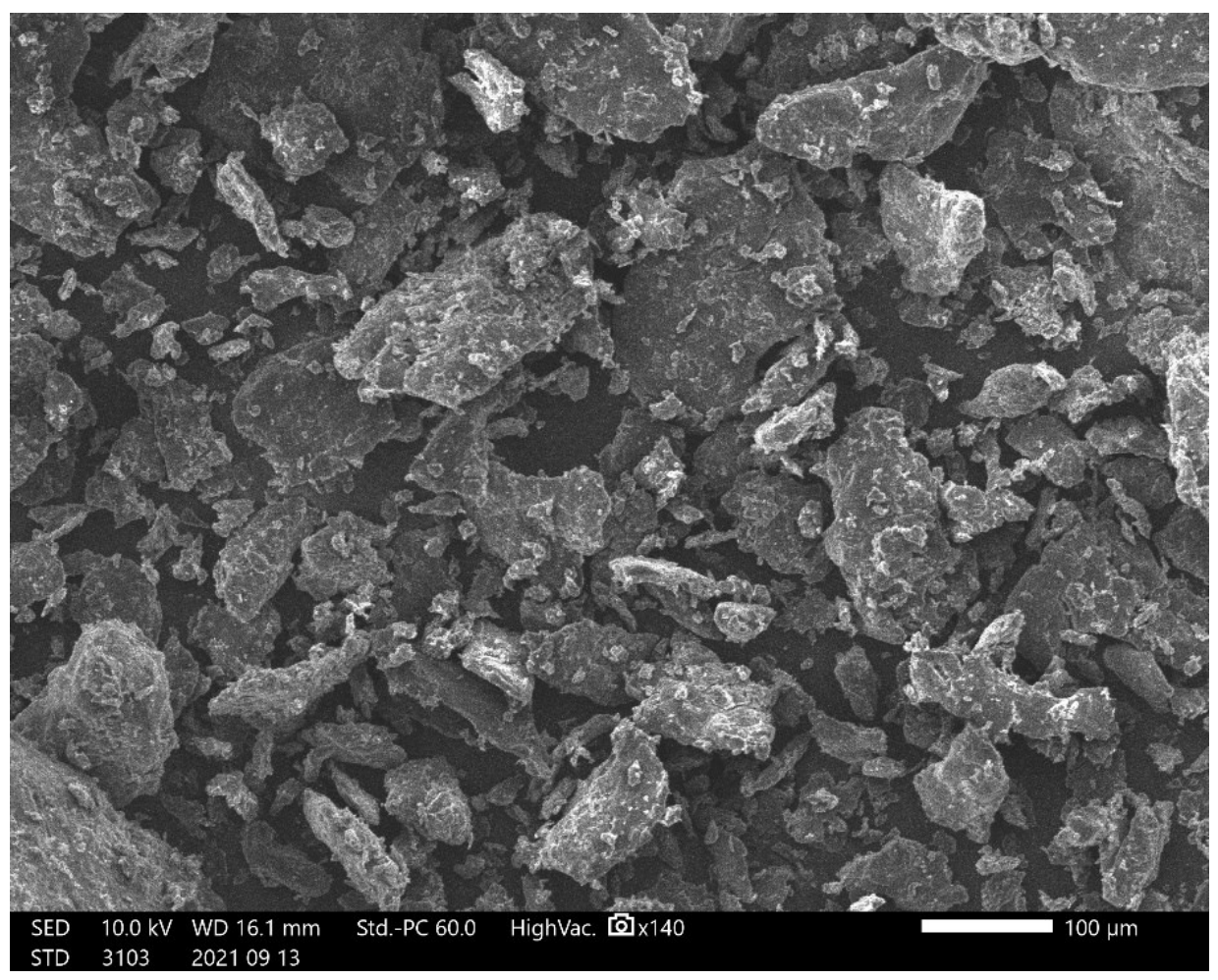

Fig. 3. SEM image of the Gelatin-cellulose Hydrogel Membrane

Figure 3 indicates the SEM analysis in the morphological structure of the Gelatin-CNCs Membrane. Porosity characterization is based on the presence of open pores which are related to properties such as permeability and surface area of the porous structure. The SEM image indicates the presence of bigger particles with irregular shapes (Fig. 3). Figure 3 also shows the cavities of different shapes and sizes, and larger pores between the particles could be observed which will be helpful for the solution permeating through the membrane [15]. The high porosity provides a favourable removal of heavy metal ions [16]. The presence of such granules increases the surface area of the composite which is suitable for effective adsorption of heavy metal ions. Small openings and holes on the surface increase the contact of the adsorption and therefore lead to pore diffusion during the removal process [17]. A morphology that changes as the gelatin concentration increases (Fig. 3) and consequently, several pores have been observed. 


\subsubsection{TGA analysis}

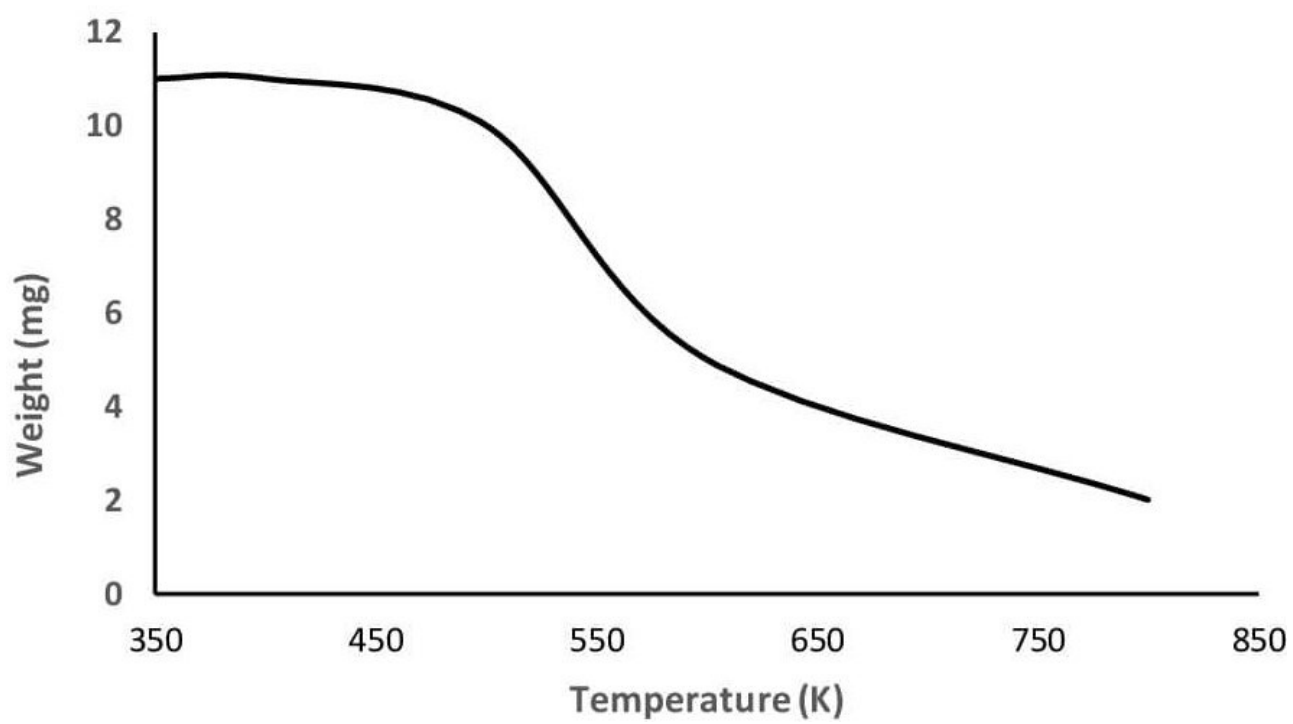

Fig. 4. TGA curve of membrane shows the relationship of weight and temperature

The characterization of the membrane was further studied by using TGA analysis. TGA analysis monitored the thermal degradation behaviour of the membrane, as a function of temperature in controlled surroundings. The TGA analysis result of membrane is shown in Figure 4. The first degradation of all films started from 350 to $800^{\circ} \mathrm{C}$ with the mass weight loss ranged from 12 to $2 \mathrm{mg}$. This observation might be due to the decomposition of highly connected amino acids group in the protein films [18]. Due to its crystalline structure, the incorporation of cellulose into the gelatin film forming solution is able to improve the thermal stability the films. The thermal decomposition of gelatin-cellulose blend films is shifted towards higher temperature as compared to gelatin films. The enhancement in thermal stability of gelatin-cellulose films can be attributed to the intermolecular and intramolecular interaction between the polymers. Overall, the results obtained from TGA analysis corroborate the earlier findings for FT-IR analysis, particularly the interaction behaviour between the polymers.

\subsection{Selection of backpropagation training algorithm and transfer function}

In this study, 10 backpropagation algorithms were compared to select the best. For all backpropagation algorithms, a 3-layer NN with tansig at hidden layer and a purelin at output layer were used. Levenberg-Marquardt (LM) backpropagation training algorithm (trainlm) with a minimum mean squared error (MSE) was found as the best. The results presented in Table 1 revealed that LM backpropagation has the smallest MSE value $(\mathrm{MSE}=0.00029)$. So LM backpropagation was considered as the training algorithm. The next factor is the transfer function, different transfer functions were examined in each layer. Among the different transfer function, log-sigmoid function was selected for all neurons due do its better prediction performance than other transfer function [19]. 
Table 1. Comparison of 10 backpropagation algorithm

\begin{tabular}{|c|c|c|c|}
\hline Backpropagation algorithm & Function & MSE & $\mathrm{R}^{2}$ \\
\hline Batch gradient descent & traingd & 0.44628 & 0.85 \\
\hline Batch gradient descent with momentum & traingdm & 0.50724 & 0.56 \\
\hline BFGS quasi-Newton backpropagation & trainbfg & 0.06100 & 0.88 \\
\hline Fletcher-Reeves conjugate gradient backpropagation & traincgf & 0.05422 & 0.82 \\
\hline Levenberg-Marquardt backpropagation & trainlm & 0.00029 & 0.94 \\
\hline One step secant backpropagation & trainoss & 0.07461 & 0.82 \\
\hline Polak-Ribiere conjugate gradient backpropagation & traincgp & 0.04871 & 0.84 \\
\hline Powell-Beale conjugate gradient backpropagation & traincgb & 0.15762 & 0.88 \\
\hline Scaled conjugate gradient backpropagation & trainscg & 0.14287 & 0.89 \\
\hline Variable learning rate backpropagation & traingdx & 0.64783 & 0.45 \\
\hline
\end{tabular}

\subsection{Neural network modelling results}

The NN model is designed to incorporate the mechanical properties of the Gelatin-CNCs membrane as input data and to predict the removal efficiency of heavy metals from wastewater.

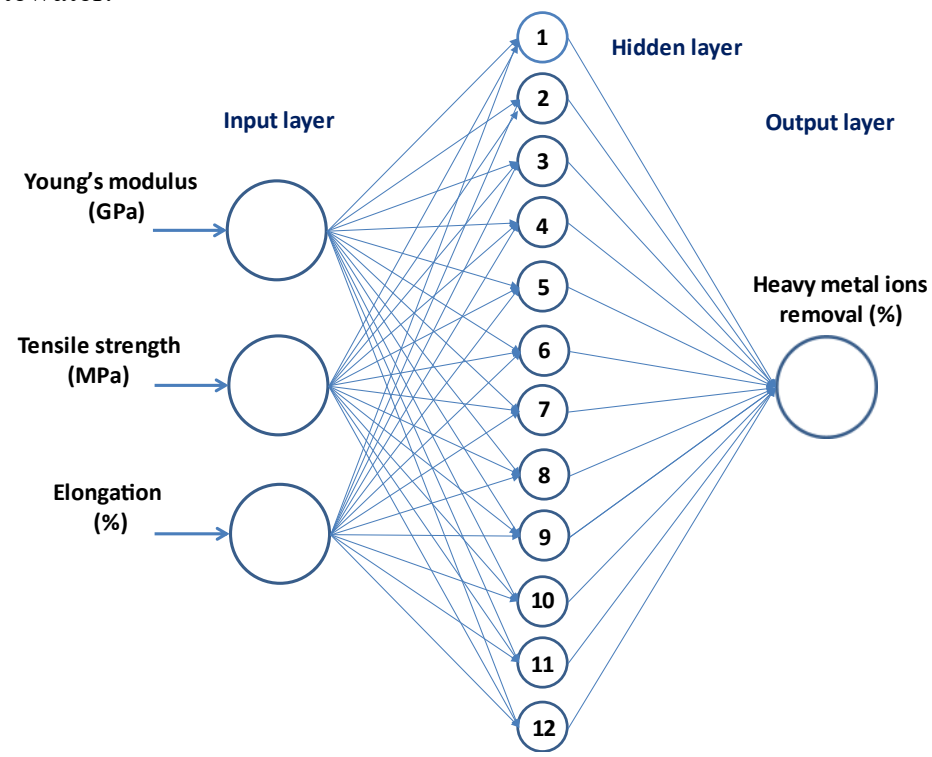

Fig. 5. Structure of Neural Network representing inputs and outputs of the model 
Figure 5 shows the structural design of the NN model with input, hidden and output layers. The structure of the NN was obtained by trying different number of hidden layers and neurons. The NN structure model (3-12-1) with a tangent sigmoid transfer function (tansig) at hidden layer with 12 neurons and a linear transfer function (purelin) at output layer was obtained as presented in Figure 5.
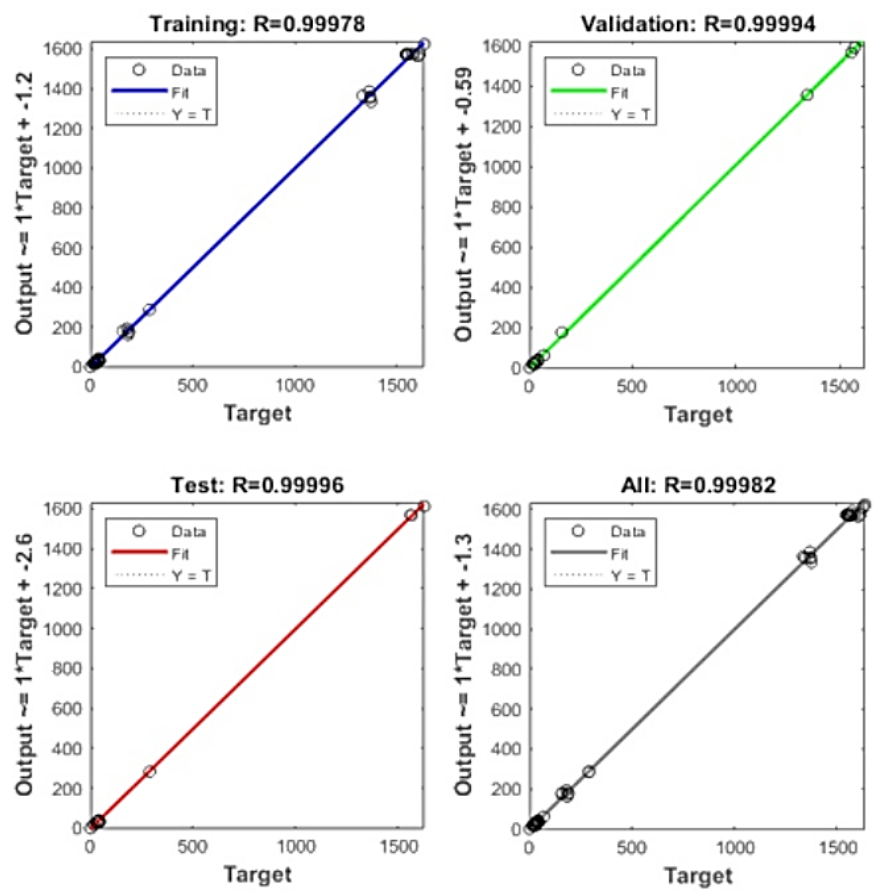

Fig. 6. Regression analysis of the network response between NN output and the corresponding targets

The scatter plots in Figure 6 illustrate a high efficiency of the neural network. Measurements of mechanical properties are based on the most probable predictions from the NN model for training, validation, testing, and overall data set. All $R^{2}$ have high values and are close to each other, which signifies the high performance of the model. For all set $\left(R^{2}=0.99315\right)$, a significant part of predictions is in excellent correlation with the experimental data. Hence, it can note that the developed $\mathrm{NN}$ is appropriate and can predict tensile and impact values accurately. The testing results indicate a good generalization capability between input and output data. Figure 6 provides a comparison of the mean values of experimental and predicted data. At the output, the developed NN model successfully predicts mechanical properties of Gelatin-CNCs membrane for the removal efficiency of heavy metals.

\subsection{Significance of the developed Neural network model}

The objective of this study is to enable to predict accurately the mechanical properties of membrane as a function of the removal of heavy metals from wastewater. However, most of the NN models developed in previous research. In this case, the measurement data are scattered and the repetition rate is low. At the same time, modelling of mechanical properties is very difficult due to the complex interaction of several parameters. These interactions are too complex and poorly understood. In the current work, a well-trained model was elaborated, which could be an excellent candidate to predict the mechanical properties as a function of heavy metals removal efficiency. NN approach model can significantly reduce the need for experimental measurement of mechanical properties, which shall be greatly appreciated in membrane technology for wastewater treatment industries. 


\subsection{Mechanical Properties}

The mechanical properties such as Young's modulus, tensile strength, and elongation of the Gelatin-cellulose hydrogel membrane were investigated with tensile tests and the results in the form of stress-strain curves shown in Figure 7. $\mathrm{pH}$ and CNCs content significantly regulate the mechanical properties of gelatin films. Gelatin-CNCs membrane for $\mathrm{pH} 4$ presented significantly increased of stress between 0 and 5\% strain. This may be attributed to the electrostatic attractive interaction between gelatin and CNCs reinforcing effect through stress transfer from the gelatin matrix to CNCs. When $\mathrm{pH}$ is 6 , the increase of stress was observed to be almost half compared to $\mathrm{pH} 4$ most likely due to the reduction in the electrostatic interactions above the gelatin. For the alkaline condition at $\mathrm{pH} 8$, the stress increases at $15 \mathrm{MPa}$ and remains constant due to the regeneration, which increases the overall crystallinity of the films. The mechanical properties behaviour of Young's modulus, tensile strength, and elongation can be attributed to the formation of agglomerates as revealed by the SEM result in Figure 3. The gelatin film with CNCs showed an elongation at break higher than that of the pure gelatin film, indicating that the gelatin-CNCs membranes were much more ductile and flexible. The interaction formed between the gelatin and CNCs weakened the protein-protein interactions, which were effective in stabilizing the gelatin network, as described in the work done by Zhuang et al. [20]. Therefore, it is clear that CNCs play an effective role in enhancing the mechanical properties of gelatin, with promising features for heavy metal ions removal from wastewater. The results agree with the works done by Leite et al., [6] for the CNCs nanocomposite films.

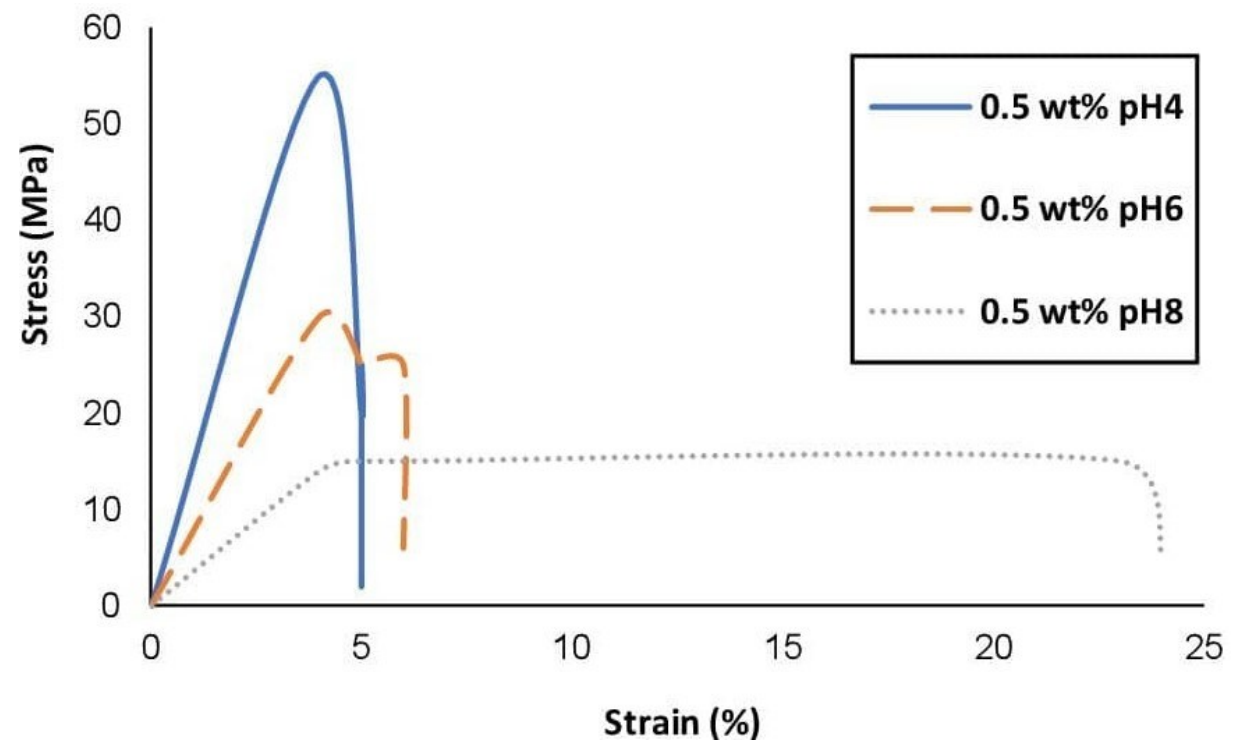

Fig. 7. Stress-strain curves of membrane film as a function of $\mathrm{pH}$ and Gelatin-CNCs membrane content

It can be seen from the Figures 8 that the maximum removal (72\%) for the tensile strength was $50 \mathrm{MPa}$; in Figure 9, the elongation was $72 \%$ at the maximum removal $(74 \%)$; and in Figure 10, the maximum removal (77\%) for the Young's modulus was $19 \mathrm{GPa}$. Figures 8-10 provide a comparison of the mean values of experimental and predicted data. At the output, the developed NN model successfully predicts various mechanical characteristics. In Figures 8-10, the results also show a slight variation for the tensile strength and elongation at break except for the Young's modulus due to the high inherent scatter of the impact properties. In terms of the relation between the experimental data and the predicted values of heavy metals removal by the NN models. Figures 8-10 show that predicted values are in good agreement with the experimental data. 


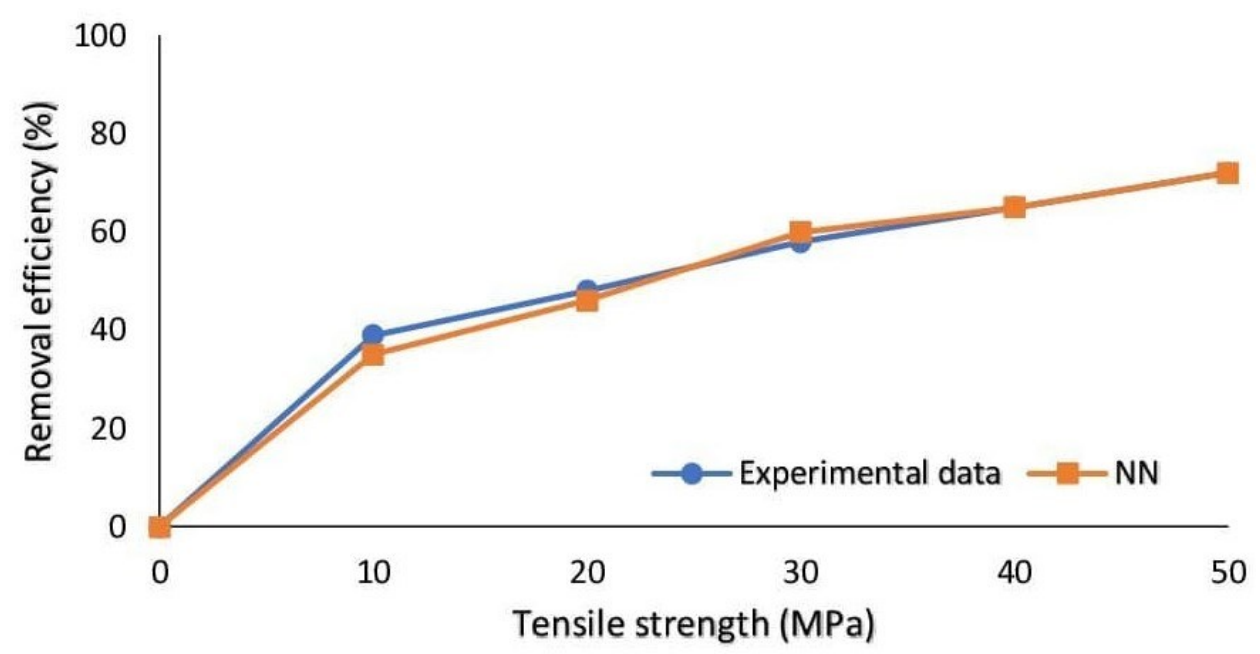

Fig. 8. Comparison between $\mathrm{NN}$ outputs and experimental data for Tensile strength at $\mathrm{pH} 4$

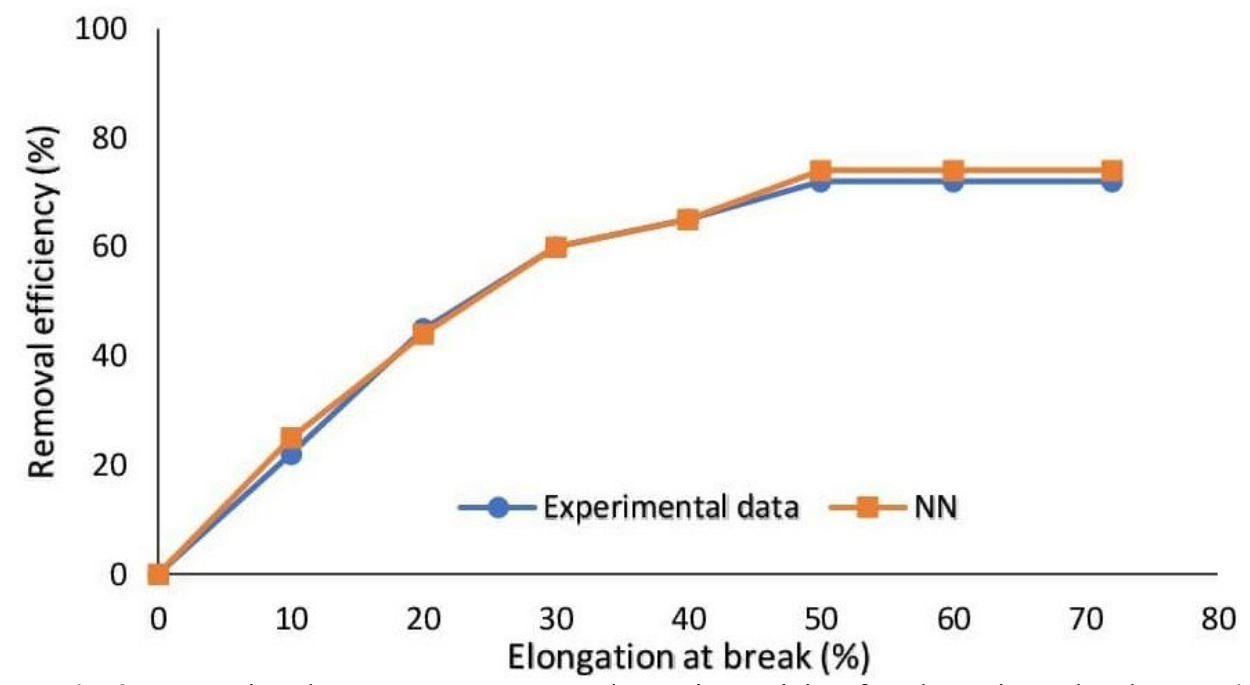

Fig. 9. Comparison between NN outputs and experimental data for Elongation at break at $\mathrm{pH} 4$

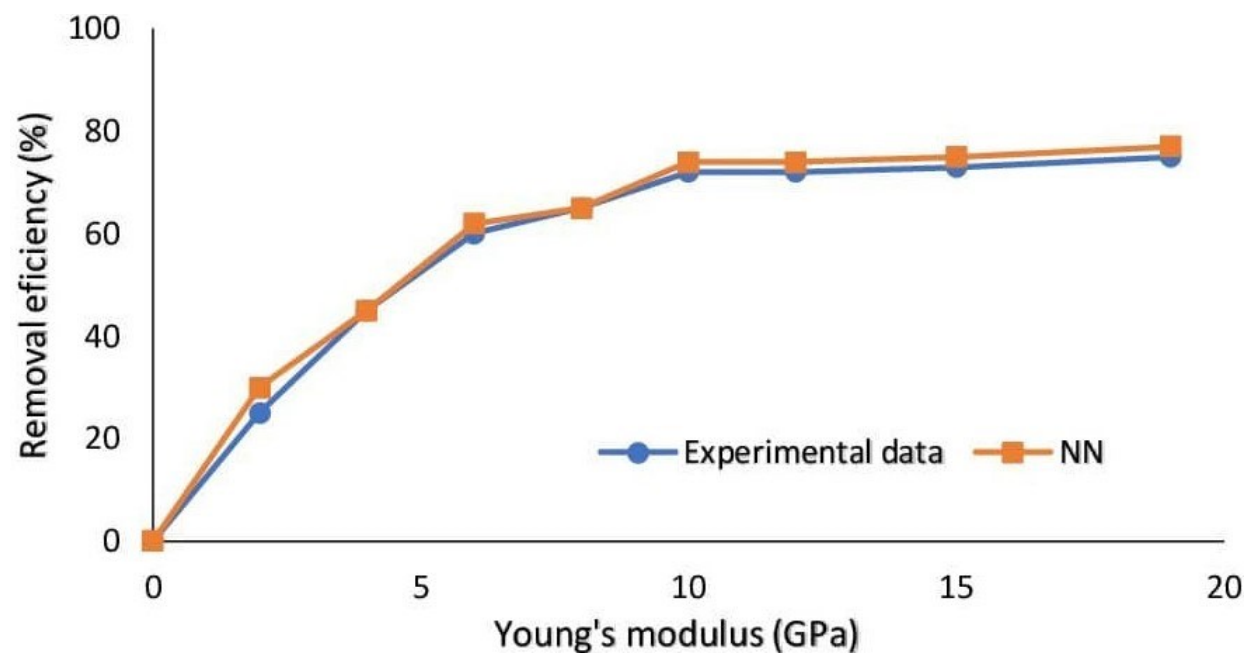

Fig. 10. Comparison between NN outputs and experimental data for Young's modulus at pH 4 


\section{Conclusion}

Neural network model was designed to predict the mechanical properties of Gelatin-cellulose hydrogel membrane. The predicted and experimental values for the present model are in a good correlation and highlight the success of the usage of NN for easier prediction of mechanical properties such as tensile strength, elongation and Young's modulus for the removal of heavy metal ions from wastewater. The configuration of the BPNN giving the smallest MSE was LMA with a tangent sigmoid transfer function at hidden layer with 12 neurons and a linear transfer function at output layer. The NN predicted results are very close to the experimental results with $R^{2}=0.99315$. The developed model possesses superior predictive capabilities as compared to the results obtained from the regression analysis. Neural networks are powerful tool that can be used to minimize the cost of experimental research.

\section{References}

1. I. García-Díaz, F.A. López, F. J. Alguacil. Metals, 8, 914 (2018)

2. J. Kabuba. Eng. Letters. 29, 158 (2021)

3. E. O. Ezugbe, S. Rathilal. Membranes, 10, 10050089 (2020)

4. R. Singh, N. Hankins. Elsevier: Amsterdam, The Netherlands (2016).

5. Z. Wang, J. Zhou, X. Wang, N. Zhang, X. Sun \& Z. Ma. Food Hydrocolloids, 35, 51 (2014).

6. L.S. F. Leite, F.K.V. Moreira, L.H.C. Mattoso, J. Bros. Food Hydrocolloids. 113, 106424 (2021)

7. S. T. Hosseini \& M. C. Gomez-Guillen. Trends in Food Sci. Technol. 79, 125 (2018)

8. M. T. Hoang, T. D. Pham, D. Verheyen, M.K. Nguyen, T.T. Pham, J. Zhu, B. Van der Bruggen, Chem. Eng. Sci. 228, 115998 (2020)

9. J. George \& Siddaramaiah. Carbohydrate Polymers, 87, 2031 (2012)

10. L. S. F. Leite, C.M. Ferreira, A. C. Correa, F.K. V. Moreira \& L. H. C. Mattoso, Carbohydrate Polymers, 238, 116198 (2020)

11. J. Kabuba, A. Mulaba Bafubiandi, K. Battle. Arab. J. Sci. Eng. 39, 6793 (2014)

12. A. Alemdar, M. Sain. (2008). Bioresource Technol. 6, 1664 - 1671 (2008)

13. P. Fernandes de Almeida, S. D. da Silva Lannes, F. Araújo Calarge, T. M. de Brito Farias, J. C. C. Santana. J Chem Chem Eng. 6, 1029 - 1032 (2012)

14. R. M. Silvestein, F. X. Webster, D. J. Kiemle, D. L. Bryce. Spectroscometric identification of Organic Compound, 8th edition. Wiley. New York, 81 - 108 (2007)

15. X. Wang, P. Liu, F. Liu, X. Wang, M. Ji, L. Song. Adsorption Sci Technol. 36, 1571 1594 (2018).

16. M. J. Hossan, M. A. Gafur, M. R. Kadir, M. M. Karim. Polymer Composites. 14, 24 32 (2014)

17. J. Kabuba. Eng. Letters. 27, 467-474 (2019)

18. M. S. Samsi, A. Kamari, S. M. Din, G. Lazar. J Food Sci Technol. 56, 3099-3108 (2019)

19. J. Kabuba and K. Aziza, Artificial Intelligence Neural Network Techniques to Predict the Removal of Anions from Wastewater, in Proceedings of the 11th South African Conference on Computational and Applied Mechanics, SACAM 2018, 17-19 September 2018, Vanderbijlpark, South Africa (2018)

20. C. Zhuang, F. Tao, Y. Cui, J. Sci. Food Agric. 97, 3384-3395 (2017) 